\title{
Detection and Localization of Spherical Markers in Photographs
}

\author{
Josip Tomurad \\ Faculty of Electrical Engineering and Computing \\ University of Zagreb \\ Zagreb, Croatia \\ e-mail: josip.tomurad@fer.hr
}

\author{
Marko Subašić \\ Faculty of Electrical Engineering and Computing \\ University of Zagreb \\ Zagreb, Croatia \\ e-mail: marko.subasic@fer.hr
}

\begin{abstract}
This paper presents two solutions for detection and localization of spherical markers in photographs. The proposed solutions enable precise detection and localization in sub millimeter range. High precision localization is required for brain surgery, and presented research effort is part of a project of developing and deploying a robotic system for neurosurgical applications. Two algorithms for Hough transform using several edge detection algorithms are proposed, and their results compared and analyzed. Results are obtained for both NIR and visible spectrum images, and required high precision is achieved in both domains.
\end{abstract}

Keywords - project RONNA, image processing, circle detection, Hough transform, edge detection

\section{INTRODUCTION}

Project RONNA (Robotic Neuronavigation) is focused in research and development of a robotic system for neurosurgical application. [1] The system consists of two robotic arms assisting the doctor in brain surgery. At the first public demonstration of the project, an operation was demonstrated in which RONNA was tasked with precise drilling of the skull. It also enabled access to the tumor in the patient's brain with accuracy of less than a millimeter. Very precise estimation of the robots position is clearly an important task in the process.

This paper is focused on the specific task of this project, detection of spherical markers that the robot uses to estimate its position in space. Detection is performed using cameras mounted on the robot. The markers are spheres coated in special paint that appears black in visible spectrum, but reflects NIR light very well. The robot is also equipped with NIR light source, which makes the markers very bright in NIR images.

This paper investigates some well-known methods of edge and circle detection in images. Two variants of the Hough transform using several methods of edge detection were selected. Used algorithms, their advantages and disadvantages, as well as other problems that have an effect on rate of detection and localization precision are described.

\section{HOUGH TRANSFORM}

Hough transform is a technique of feature extraction that is used in image analysis, computer vision, and digital image processing. The purpose of this technique is finding imperfect instances of objects from a certain class of shapes by application of voting. The technique was originally used for line detection in images, and was later expanded to recognition of various kinds of shapes, most commonly ellipses and circles.

Hough transform uses shape edges as its input so first step is finding edge pixels in an image. Then each edge pixel votes in a Hough parameter space in a pattern that describes potential shape of interest. Finally, local maximums in the parameter space provide detection candidates.

Our goal is detection of spherical objects in 2D images so our shape of interest is a circle. In a two-dimensional space, a circle is described by:

$$
(x-a)^{2}+(y-b)^{2}=r^{2},
$$

where $(a, b)$ are coordinates of the circle center, and $r$ the radius. For a given edge point with coordinates $(x, y)$ all possible combinations of parameters $a, b$ and $r$ can be found using the equation (1). In this case, possible parameter combinations lie on the surface of an inverted right-angled cone whose apex is at $(x, y, 0)$. In a 3-D Hough parameter space a circle is defined by an intersection of many conic surfaces. The points where those intersections are located are local maximums in the accumulation matrix representing the parameter space. [2]
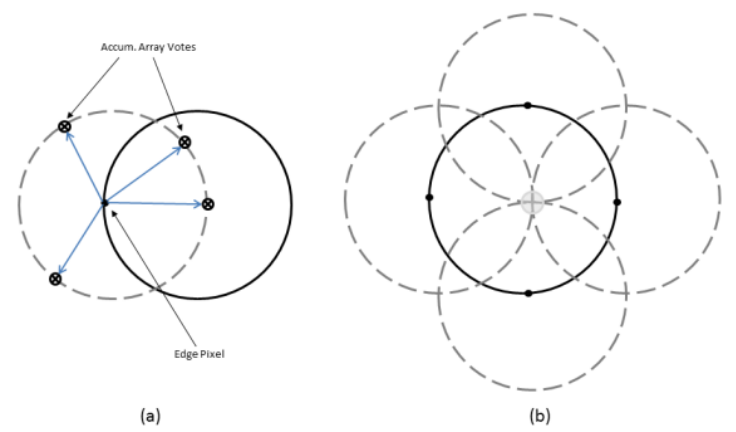

Figure 1. The voting process in the Hough transform [3] 
This algorithm is implemented by iterating trough the expected radius range and voting in the accumulation matrix for every edge pixel in the image. Every edge pixel $(x, y)$ increases value (gives vote to) of all matrix cells at coordinates $(a, b, r)$, where $r$ is fixed, and $a$ and $b$ are calculated by using equation (1). Votes for center candidates for selected radius are distributed in a circular pattern designated by the dashed line in Figure 1 (a). Figure 1 (b) shows detected center as a common intersection of these circular patterns originating from several edge points. Each detected center is a local maximum in the accumulation matrix and it needs to be above Hough accumulation threshold in order to be considered.

The accumulation matrix in the classic implementation of the Hough transform is tri-dimensional. Its dimensions are $n, m, p$, where $n$ and $m$ are image dimensions, and $p$ is the size of radius range within which the circle is searched for. Because this demands a large amount of memory and a long processing time, it is common in modern practice to use a two-dimensional matrix, of dimensions $n, m$. In this case, only the location of the center is calculated, and this process demands an extra step of radius evaluation. The approach is less computationally demanding, especially if a larger radius range is used. In this paper both approaches are tested.

\section{EDGE DETECTION}

Hough transform uses object edges as its inputs, and there are many ways to detect them. Sobel operator, alone and in combination with the Gauss filter, as well as Canny edge detector, and Laplacian of Gaussian (LoG) were used in presented research. These methods differ in the way they calculate edge pixels, which entails different complexity, and gives different results. Relevant parameter for all methods is edge detection threshold, the minimum value of gradient a pixel must have to be considered part of an edge.

The Sobel operator works on a principle of gradient estimation in a digital image by summation of vectors of two orthogonal gradient estimations that can be calculated from a $3 \times 3$ neighborhood of a point. $[4,5,6]$

Canny edge detection is an edge detection operator that uses a multi-stage algorithm to detect a wide range of edges in an image. It provides very good and reliable detection. [7]

When searching for edges by using LoG, the image is first convolved by a Gaussian filter, in order to minimize noise. Subsequently, the Laplacian operator is applied to the image. [8] This method is also very precise and reliable, comparable to the Canny algorithm.

\section{METHODS}

\section{A. Hough transform with radius estimation}

We utilized the Two-Stage method developed by Yuen et al. [9] and discussed by Davies. [10] This method works by utilizing voting of edge pixels to find the center of the circle, and the radius is estimated afterwards. This estimation is based on radial histograms. A 2-D accumulation matrix is used, with the benefit of a significant performance increase relative to a $3-\mathrm{D}$ matrix. In order to optimize performance, information about edge gradient is used to enable voting only along a limited interval in the gradient direction. [11] Consequently this method requires edge detection methods that provide gradient angle. Edge detection can be preceded by Gauss smoothing, in order to reduce the number of detected pixels that are not actual edges.

Variable parameters of this method are the range of radii in which we circles are searched for, edge detection threshold, and Hough accumulation threshold.

\section{B. Hough transform using a 3-D accumulation matrix}

The first step of this method is edge detection using one of previously described methods. Then Hough transform is applied to the binary image, by first using midpoint circle algorithm [12] to calculate circle templates of all the radii in the relevant range. Those templates determine which accumulator elements need to be incremented relatively to the current pixel. Then comes the voting, done by iterating the calculated patterns through all the edge pixels and incrementing the appropriate elements of the accumulation matrix.

The next step is searching for as many local maximums in the accumulation matrix as there are circles we are searching for in the image. Neighborhood suppression is applied, meaning that after a particular maximum is found, its immediate neighborhood is no longer taken into consideration. This is due to the large chance that other local maximums will be found adjacent to the first one, but all of them are probably a consequence of the same circle.

The coordinates of local maximums are actual center positions and radii of detected circles. This method can be optimized by not searching for a particular number of circles, but setting a Hough accumulation. This is useful in when we don't know the number of circles in an image is unknown.

Variable parameters in this method are radius range, sigma (standard deviation of the Gauss distribution), and edge detection threshold.

\section{RESULTS}

MATLAB was used as the development environment in this paper.

The key feature in presented result tables is the deviation of center and radius estimation relative to the manually estimated gold standard ("Center delta" and "Radius delta" in the tables). Manual estimation was done by hand picking 6 points, and calculating the mean of all 20 possible circles those points define. 
Infrared images of dimensions $1280 \times 1024$ pixels, and visible spectrum images of 2590x1942 pixels were used in experiments. NIR images are processed in the original size, while VS images are reduced to half the original size in order to speed up the process. Our database has 19 NIR and 20 VS images. The markers are $10.9 \mathrm{~mm}$ in diameter.

The processing times reported in following subsections are very dependent of the performance capabilities of the computer system used, but it can be used as a framework for comparison of different methods.

\section{A. Hough transform with radius estimation}

Radius range for NIR images is [95, 170], edge detection threshold is 0.1, and Hough accumulation threshold is 0.94 .

Radius range for VS images is [250, 300], edge detection threshold is 0.1 , and Hough accumulation threshold is 0.97 .

Two variants were tested, the first with no preprocessing designated as Method (1) in Table 1 and the second with preprocessing using the Gauss filter (2) designated as Method (2) with sigma being set to 2 . Both methods use the Sobel operator to find the edge pixels.

These two methods are a bit more imprecise compared to those using the 3-D accumulation matrix, but are significantly faster. Using the 2-D matrix reduces processing time to 1-2 seconds per image. IR images are relatively easily processed because of high contrast between the marker and background. That makes the edges easily detectable (Figure 2).

Table 1. Results of Hough transform with radius estimation

\begin{tabular}{|c|c|c|c|c|}
\hline \multicolumn{5}{|c|}{ Method (1) } \\
\hline Image type & \multicolumn{2}{|c|}{ Infrared } & \multicolumn{2}{|c|}{ Visible spectrum } \\
\hline & $\begin{array}{c}\text { Center } \\
\text { delta }\end{array}$ & $\begin{array}{c}\text { Radius } \\
\text { delta }\end{array}$ & $\begin{array}{c}\text { Center } \\
\text { delta }\end{array}$ & $\begin{array}{c}\text { Radius } \\
\text { delta }\end{array}$ \\
\hline mean $[\mathrm{px}]$ & 3.14 & 2.32 & 5.21 & 3.71 \\
\hline mean $[\mathrm{mm}]$ & 0.106 & 0.078 & 0.100 & 0.071 \\
\hline std. dev. [px] & 2.17 & 1.08 & 5.30 & 3.21 \\
\hline std. dev. [mm] & 0.073 & 0.036 & 0.101 & 0.061 \\
\hline \multicolumn{5}{|c|}{ Method (2) } \\
\hline Image type & \multicolumn{2}{|c|}{ Infrared } & \multicolumn{2}{|c|}{ Visible spectrum } \\
\hline & $\begin{array}{c}\text { Center } \\
\text { delta }\end{array}$ & $\begin{array}{c}\text { Radius } \\
\text { delta }\end{array}$ & $\begin{array}{c}\text { Center } \\
\text { delta }\end{array}$ & $\begin{array}{c}\text { Radius } \\
\text { delta }\end{array}$ \\
\hline mean $[\mathrm{px}]$ & 2.09 & 1.11 & 7.02 & 4.29 \\
\hline mean $[\mathrm{mm}]$ & 0.070 & 0.037 & 0.134 & 0.082 \\
\hline std. dev. [px] & 1.14 & 0.85 & 6.20 & 3.69 \\
\hline std. dev. $[\mathrm{mm}]$ & 0.038 & 0.029 & 0.118 & 0.071 \\
\hline
\end{tabular}

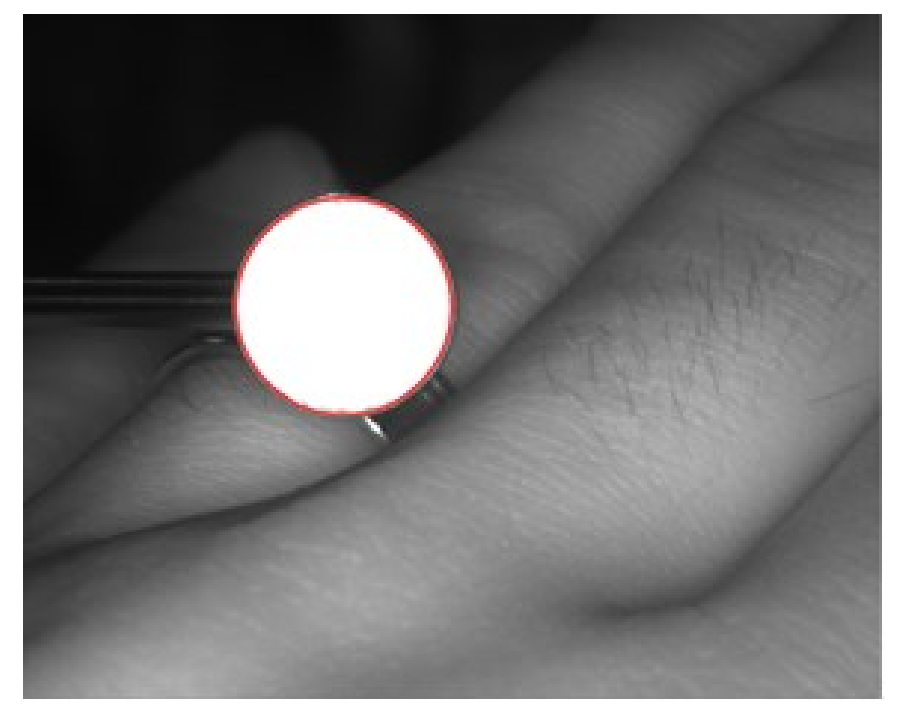

Figure 2. Circle detection by method (1) in an NIR image

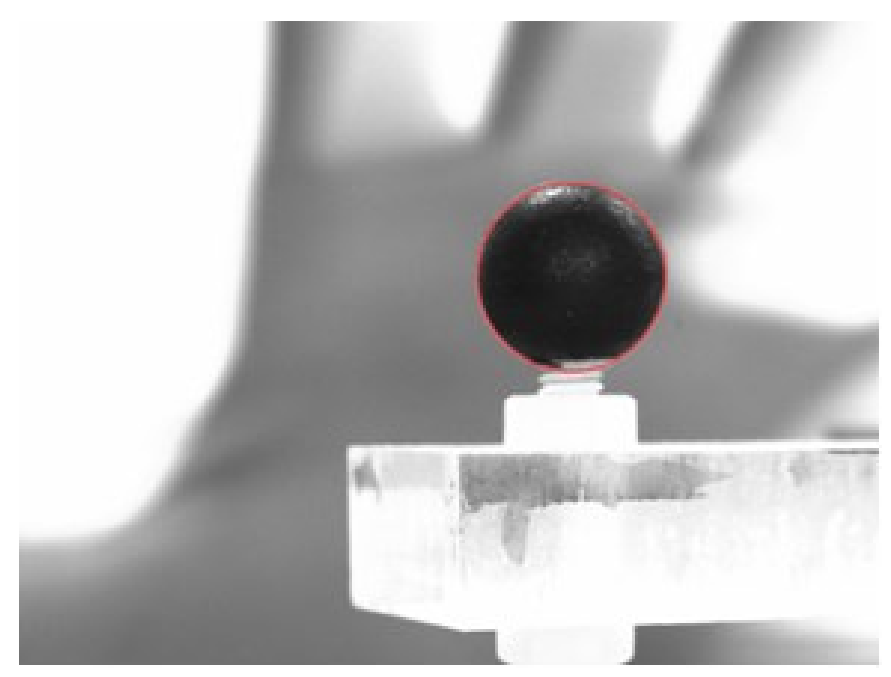

Figure 3. Circle detection by method (2) in an VS image

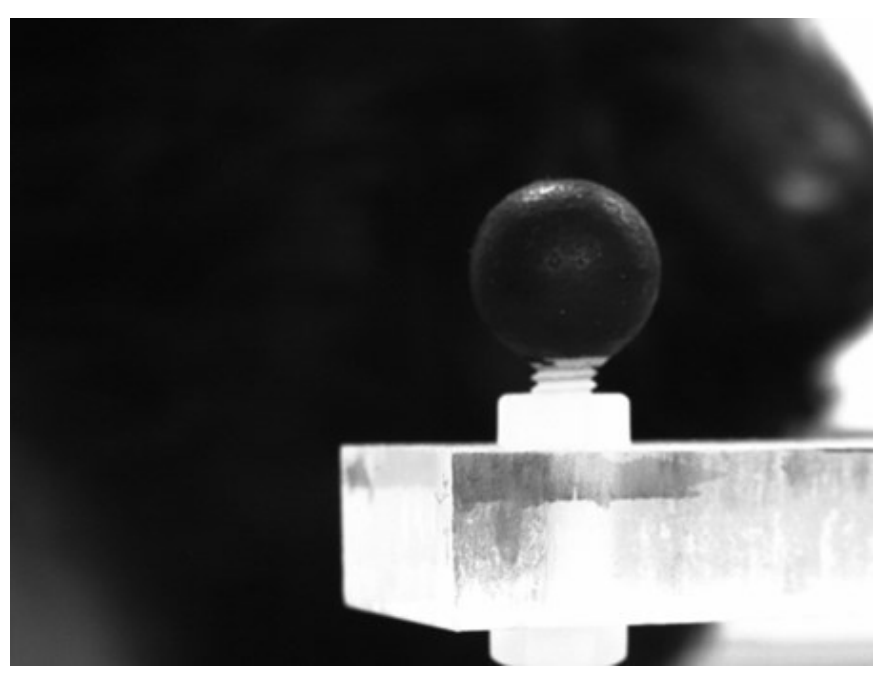

Figure 4. VS, low contrast between the marker and background (method (1)) 
Some VS images are also suitable for circle detection (Figure 3). However, these images have less distinct marker edges, leading to complete inability to detect circles in some of them (Figure 4) or imprecise detection (Figure 5). Additionally, Hough accumulation threshold has to be set quite low when processing VS images, due to the low number of edge pixels, which often leads to false detection (Figure 5).

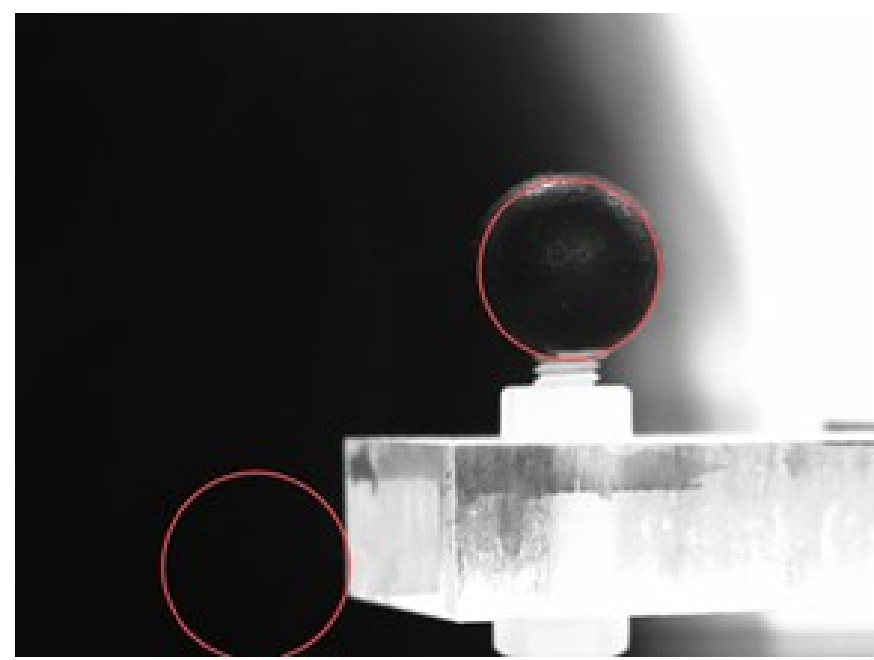

Figure 5. VS, detection by method (2)

\section{B. Hough transform using a 3-D accumulation matrix}

Four variants of this method are proposed and tested, depending on the edge detection operator used. Neighborhood suppression parameters are 51 pixels in all 3 directions. Method (3) uses the Sobel operator (the same as method (1))

Method (4) uses the Sobel operator and Gauss filter preprocessing with the parameter sigma 2 (same as method (2)).

Method (5) utilizes Canny edge detection.

Method (6) uses the Laplacian of Gaussian

The IR images edge detection threshold is 0.2 and Gauss smoothing parameter sigma is 5 .

The VS images edge detection threshold is 0.00007 and Gauss smoothing sigma is 8 .

As stated previously, these methods take longer to execute, but the results are slightly better. Processing time for an image is on the order of 20-30 seconds. IR images give better results (Figure 6) than VS images (Figure 7) here as well, although the difference is reduced.

Methods (3) and (4) can be directly compared to methods (1) and (2) because of the same way of finding edges. Results show that calculation using a 3-D matrix is more precise than the one with a 2-D matrix. Another advantage of this approach is improved circle detection in low-contrast images. Figure 8 shows such an example where method (3) successfully detects the circle, while method (1) is unsuccessful.

Methods (5) and (6) utilize more advanced edge detection techniques, and are comparable precision-wise to methods (3) and (4). Canny and LoG additionally provide a smaller number of edge pixels, due to their precision and noise reduction, reducing processing time. However, not even these methods can guarantee absolute precision in lowcontrast conditions (Figure 9).

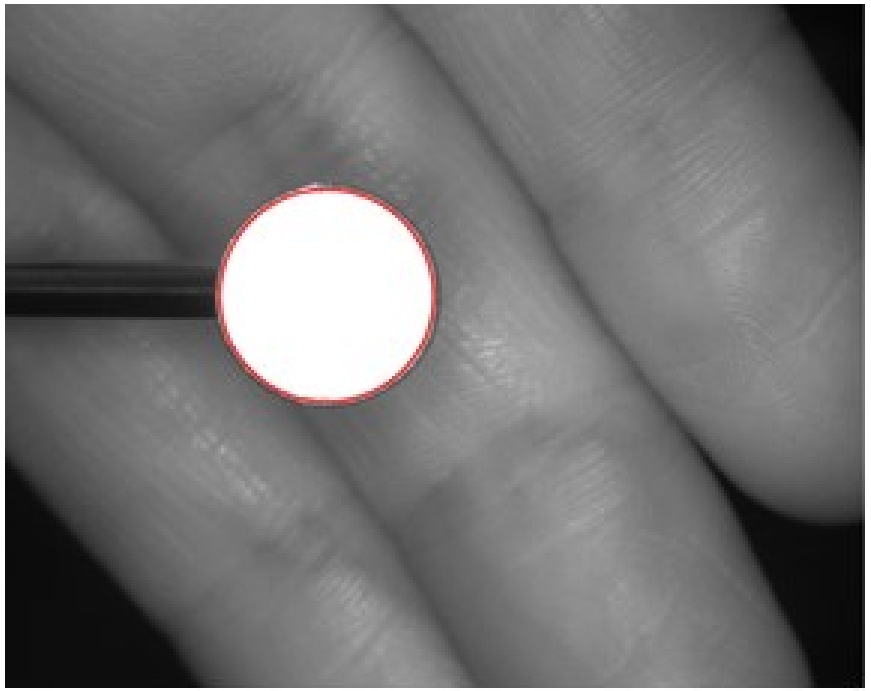

Figure 6. Circle detection using method (6) in an IR image

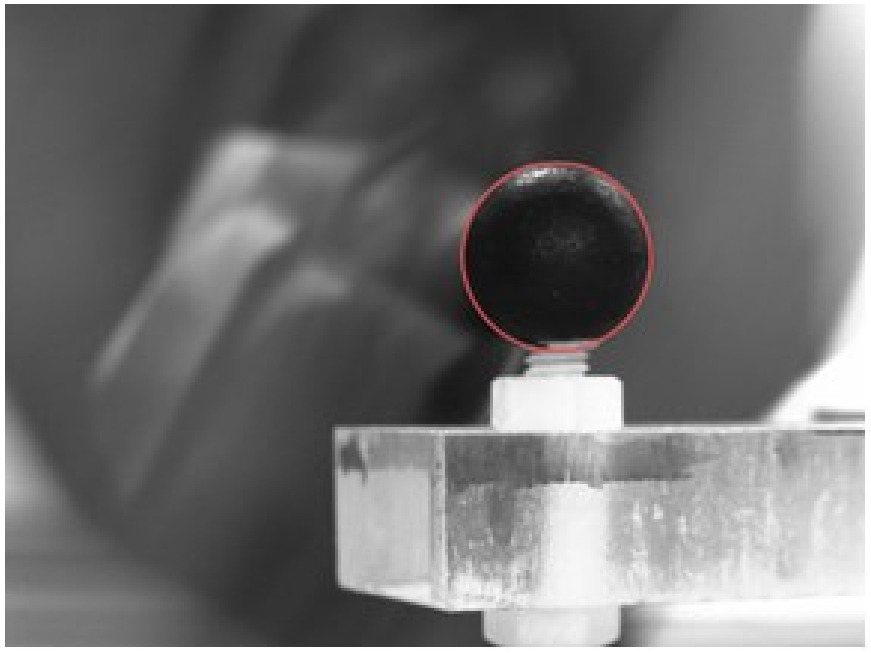

Figure 7. Circle detection using method (5) in a VS image

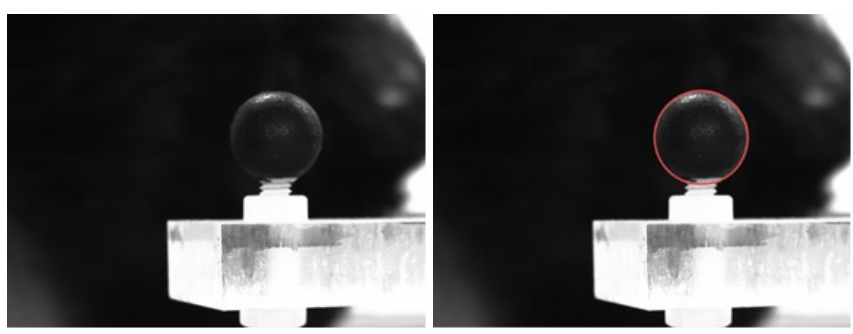

Figure 8. VS, method (1) left, method (3) right 
Table 2. Results of Hough transform using

a 3-D accumulation matrix

\begin{tabular}{|c|c|c|c|c|}
\hline \multicolumn{5}{|c|}{ Method (3) } \\
\hline \multirow[t]{2}{*}{ Image type } & \multicolumn{2}{|c|}{ Infrared } & \multicolumn{2}{|c|}{ Visible spectrum } \\
\hline & $\begin{array}{l}\text { Center } \\
\text { delta }\end{array}$ & $\begin{array}{c}\text { Radius } \\
\text { delta }\end{array}$ & $\begin{array}{l}\text { Center } \\
\text { delta }\end{array}$ & $\begin{array}{l}\text { Radius } \\
\text { delta }\end{array}$ \\
\hline mean $[\mathrm{px}]$ & 2.67 & 2.95 & 3.63 & 2.68 \\
\hline mean $[\mathrm{mm}]$ & 0.090 & 0.099 & 0.069 & 0.051 \\
\hline std. dev. [px] & 1.51 & 1.00 & 3.02 & 3.54 \\
\hline std. dev. [mm] & 0.051 & 0.034 & 0.058 & 0.068 \\
\hline \multicolumn{5}{|c|}{ Method (4) } \\
\hline \multirow[t]{2}{*}{ Image type } & \multicolumn{2}{|c|}{ Infrared } & \multicolumn{2}{|c|}{ Visible spectrum } \\
\hline & $\begin{array}{c}\text { Center } \\
\text { delta }\end{array}$ & $\begin{array}{c}\text { Radius } \\
\text { delta }\end{array}$ & $\begin{array}{c}\text { Center } \\
\text { delta }\end{array}$ & $\begin{array}{c}\text { Radius } \\
\text { delta }\end{array}$ \\
\hline mean $[\mathrm{px}]$ & 1.74 & 2.32 & 3.1 & 2.50 \\
\hline mean $[\mathrm{mm}]$ & 0.059 & 0.078 & 0.075 & 0.048 \\
\hline std. dev. [px] & 0.93 & 0.86 & 3.12 & 2.58 \\
\hline std. dev. [mm] & 0.031 & 0.029 & 0.060 & 0.049 \\
\hline \multicolumn{5}{|c|}{ Method (5) } \\
\hline \multirow[t]{2}{*}{ Image type } & \multicolumn{2}{|c|}{ Infrared } & \multicolumn{2}{|c|}{ Visible spectrum } \\
\hline & $\begin{array}{l}\text { Center } \\
\text { delta }\end{array}$ & $\begin{array}{c}\text { Radius } \\
\text { delta }\end{array}$ & $\begin{array}{c}\text { Center } \\
\text { delta }\end{array}$ & $\begin{array}{c}\text { Radius } \\
\text { delta }\end{array}$ \\
\hline mean $[\mathrm{px}]$ & 1.21 & 1.26 & 3.98 & 3.40 \\
\hline mean $[\mathrm{mm}]$ & 0.041 & 0.042 & 0.076 & 0.065 \\
\hline std. dev. [px] & 0.41 & 0.44 & 3.85 & 2.71 \\
\hline std. dev. [mm] & 0.014 & 0.015 & 0.074 & 0.052 \\
\hline \multicolumn{5}{|c|}{ Method (6) } \\
\hline \multirow[t]{2}{*}{ Image type } & \multicolumn{2}{|c|}{ Infrared } & \multicolumn{2}{|c|}{ Visible spectrum } \\
\hline & $\begin{array}{l}\text { Center } \\
\text { delta }\end{array}$ & $\begin{array}{c}\text { Radius } \\
\text { delta }\end{array}$ & $\begin{array}{c}\text { Center } \\
\text { delta }\end{array}$ & $\begin{array}{c}\text { Radius } \\
\text { delta }\end{array}$ \\
\hline mean $[\mathrm{px}]$ & 1.23 & 1.11 & 4.83 & 2.70 \\
\hline mean $[\mathrm{mm}]$ & 0.041 & 0.037 & 0.092 & 0.052 \\
\hline std. dev. [px] & 0.52 & 0.55 & 3.99 & 2.51 \\
\hline std. dev. [mm] & 0.018 & 0.019 & 0.076 & 0.048 \\
\hline
\end{tabular}

\section{CONCLUSION}

Solutions proposed in this paper yielded very precise circle localization and radius estimation. However, the precision depends largely on the quality of the processed image, its background and lighting. This is especially evident in visible spectrum images. They give good results with sufficient contrast between the marker and background, but the precision diminishes along with the reduction in

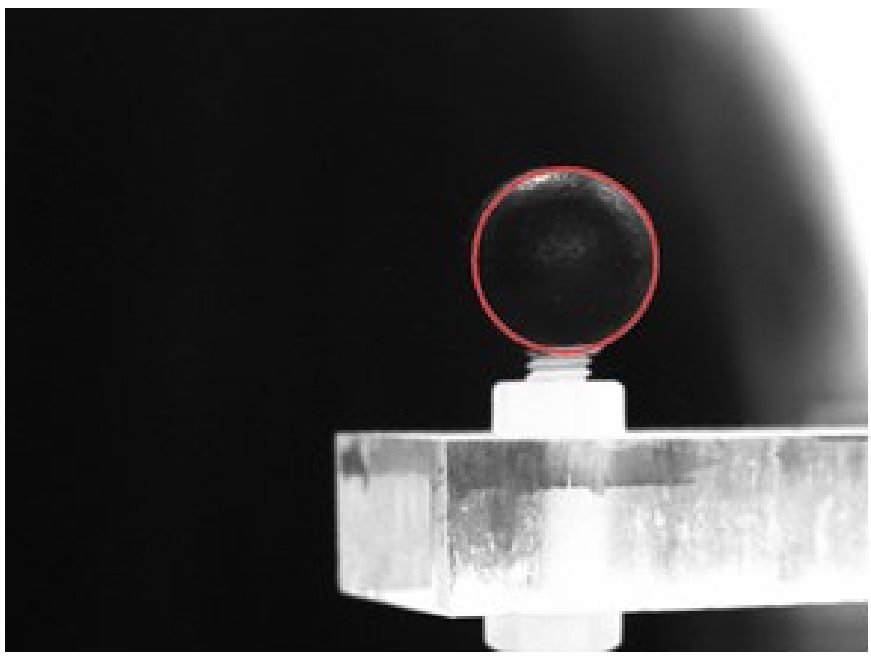

Figure 9. VS, method (5)

contrast. It is also evident that IR images yield better results than VS images, also due to higher contrast.

The Hough transform is an excellent and highly flexible tool for circle detection. In future work it is possible to research other ways of its implementation, in order to increase flexibility and robustness of circle detection.

The usage of Hough transform with a 3-D accumulation matrix yields the most precise results in both kinds of images, but this comes at the price of higher complexity. Since speed is sometimes equally as important as precision in practical circle detection applications, the fact has to be considered when choosing the optimal solution.

\section{REFERENCES}

[1] RONNA, http://www.ronna-eu.fsb.hr, 15. 6. 2016.

[2] Circle Hough Transform, https://en.wikipedia.org/wiki/Circle Hough_Transform, 15. 6. 2016

[3] Find circles using circular Hough transform - MATLAB imfindcircles, http://www.mathworks.com/help/images/ref/imfind circles.html, 15. 6. 2016

[4] Sobel, Irwin. History and Definition of the so-called „Sobel Operator", 2015

[5] Sobel Operator, https://en.wikipedia.org /wiki/Sobel_opera tor, 15. 6. 2016

[6] Feature Detectors - Sobel Edge Detector, http://homepages.inf.ed.ac.uk/rbf/HIPR2/ sobel.htm, 15. 6. 2016

[7] Canny edge detector, https://en.wikipedia. org/wiki/Canny_edge detector, 15. 6. 2016

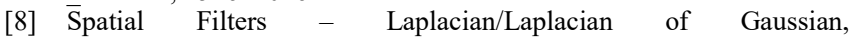
http://homepages.inf.ed.ac.uk/rbf/ HIPR2/log.htm, 15. 6. 2016

[9] Yuen, H.K., Princen, J., Illingworth, J., and Kittler, J. Comparative study of Hough transform methods for circle finding. Image and Vision Computing. Volume 8, Number 1, 1990, pp. 71-77.

[10] [Davies, E.R. Machine Vision: Theory, Algorithms, Practicalities. Chapter 10. 3rd Edition. Morgan Kauffman Publishers, 2005

[11] Atherton, T.J., Kerbyson, D.J. "Size invariant circle detection." Image and Vision Computing. Volume 17, Number 11, 1999, pp. 795-803.

[12] Midpoint Circle Algorithm, https://en.wiki pedia.org/wiki/Midpoint_circle_algorithm, 15.6. 2016 